\title{
Effect of surfactants on pressure-sensitivity of CNT filled cement mortar composites
}

\author{
Baoguo Han ${ }^{1}$ and Xun $\mathrm{Yu}^{2 *}$ \\ ' School of Civil Engineering, Dalian University of Technology, Dalian, China \\ ${ }^{2}$ Department of Mechanical and Energy Engineering, University of North Texas, Denton, TX, USA
}

\section{Edited by:}

Weihua Li, University of Wollongong, Australia

\section{Reviewed by:}

Xufeng Dong, Dalian University of Technology, China

Yin Jianbo, Northwestern

Polytechnical University, China

${ }^{*}$ Correspondence:

Xun Yu, Department of Mechanical and Energy Engineering, University of North Texas, Denton, TX 76203, USA

e-mail:Xun.Yu@unt.edu
Sodium dodecyl sulfate (SDS) and sodium dodecylbenzene sulfonate (NaDDBS) were used as surfactants to disperse multi-walled carbon nanotubes (MWNT) in cement mortar and fabricate pressure-sensitive carbon nanotubes filled cement mortar composites. The pressure-sensitivity of cement mortar composites with different concentrations of MWNT and different surfactants was explored under repeated loading and impulsive loading, respectively. Experimental results indicate that the response of the electrical resistance of composites with NaDDBS to external force is more stable and sensitive than that of composites with SDS. Therefore, NaDDBS has higher efficiency than SDS for the dispersion of MWNT in cement mortar, and it is an effective surfactant for fabricating MWNT filled cement mortar composites with superior pressure-sensitivity.

Keywords: cement mortar, carbon nanotubes, pressure-sensitivity, compression, surfactant impacts

\section{INTRODUCTION}

Cement-based materials (including cement paste, cement mortar, and concrete) have long been used as structural materials for construction. Conductive fillers can be incorporated as a second phase into the matrix to produce cement-based composites with electrical conductivity (Hou and Lynch, 2005; Ou and Han, 2009). If the electrical conductivity of such composites could vary with external stress or deformation, i.e., the materials have pressure-sensitivity, the structure itself thus becomes a multifunctional structure with sensing capability to detect levels of structural stress and monitor its own structural health (Chung, 2002; Han et al., 2009a; Ou and Han, 2009; Yu and Kwon, 2009).

The pressure-sensitivity of cement-based composites was firstly investigated by Chung et al. with the findings of pressuresensitivity of cement-based composites with short carbon fibers (Chung, 2002). Since then, much work has been done on the pressure-sensitivity of cement-based composites with other electrically conductive fillers, such as carbon coated nylon fiber, steel fiber, carbon black, steel slag, and nickel powder (Mao et al., 1996; Chung, 2002; Meyyappan, 2005; Han and Ou, 2007; Han et al., 2009a). Carbon nanotubes (CNT), being the strongest known fiber and possessing excellent electrical properties, are promising candidates as the functional fillers for the next generation of pressuresensitive composites. Much previous research efforts have been concentrated on CNT filled polymer composites (Meyyappan, 2005). However, there could be some profound application of CNT in cement-based materials for infrastructure applications, which also deserves extensive scientific investigation.

Since CNTs tend to aggregates together due to the van-der Waals force, their disaggregation and uniform dispersion are critical challenges to successfully fabricating CNT filled composites. Water is the most common raw material for fabricating cement-based materials. It is critical is to produce a homogeneous dispersed suspension of CNT in water before $\mathrm{CNT}$ are added into cement-based materials. There are two major approaches to disperse CNT in water: covalent surface modification method and non-covalent surface modification method. Covalent surface modification uses strong acids at high temperatures to introduce $-\mathrm{COOH}$ groups on CNT surface, but might introduce structural defects as well and is not practical for large-scale applications. The non-covalent surface modification method use surfactants to disperse CNT in water, thus is more attractive in civil applications. Sodium dodecyl sulfate (SDS) is one of the most widely used surfactants for dispersing CNT in water. Sodium dodecylbenzene sulfonate (NaDDBS) has also been proved to be efficient in solubilizing high weight fraction single-walled CNT in water (Islam et al., 2003; Tan and Resasco, 2005; Vaisman et al., 2006; Li et al., 2007; Yu et al., 2007). However, no report is found on comparing the dispersion capability of SDS and NaDDBS to CNT in cement mortar for fabricating pressure-sensitive CNT filled cement-based composites.

In this paper, SDS and NaDDBS are used as surfactants to improve the dispersion of multi-walled CNT (MWNT) in cement mortar and fabricate pressure-sensitive CNT filled cement mortar composites. The pressure-sensitivity of CNT filled cement mortar composites with different concentrations of MWNT and different surfactants is investigated under repeated loading and impulsive loading, respectively.

\section{MATERIALS AND METHODS \\ MATERIALS}

The cement used is ASTM Type I Portland cement. The sand used is commercial grade fine sand provided by Quikrete International Inc., USA. The MWNT used are carboxyl MWNT provided by Timesnano (Chengdu Organic Chemicals Co. Ltd. of Chinese Academy of Sciences, China). Their properties are given in Table $\mathbf{1}$. The carboxyl MWNTs have a relative small percentage of $-\mathrm{COOH}$ groups (about 3.8\%), which could not induce enough negative charges on the surfaces of MWNTs. Therefore, surfactants are 
needed to stably disperse the MWNTs in water. The surfactants, SDS, and NaDDBS, are from Sigma-Aldrich Co., USA. Tributyl phosphate (Sigma-Aldrich Co., USA) was used as defoamer to decrease the air bubble in CNT filled cement mortar composites caused by use of SDS and NaDDBS.

\section{SPECIMEN PREPARATION}

Some researches have proved that the ratio of surfactant to CNT can increase the dispersing capability of CNT (Islam et al., 2003; Tan and Resasco, 2005; Vaisman et al., 2006; Yu et al., 2007), but an excessive concentration of surfactants will impair the hydration of cement and will cause a marked air-entraining effect. The $8.1 \times 10^{-3}$ and $1.4 \times 10^{-2} \mathrm{~mol} / \mathrm{L}$ of the critical micelle concentrations therefore were taken as the input surfactant concentration of SDS and NaDDBS in water, respectively. The surfactant was firstly mixed with water (the water/cement ratio is $0.46: 1$ ) using a magnetism stirrer (PC-210, Corning Inc., USA) for $3 \mathrm{~min}$. Next, MWNT ( 1 or $0.2 \%$ by weight of cement) were added into this aqueous solution and sonicated with an ultrasonicator (2510, Branson Ultrasonic Co., USA) for $2 \mathrm{~h}$ to make a uniformly dispersed suspension. Then, a mortar mixer was used to mix this suspension, cement, and sand (the ratio of sand to cement is $1.5: 1$ ) for about $3 \mathrm{~min}$. Finally, a defoamer in the amount of $0.25 \mathrm{vol} . \%$ was added into the mixture and mixed for another $3 \mathrm{~min}$.

After pouring the mixes into oiled molds $(5.08 \mathrm{~cm} \times 5.08 \mathrm{~cm} \times$ $5.08 \mathrm{~cm}$ ) and embedding two stainless steel gauzes (with opening of $1.25 \mathrm{~cm} \times 1.25 \mathrm{~cm}$ ) as two electrodes with $1 \mathrm{~cm}$ apart, an electric

Table 1 | Properties of carboxyl multi-wall carbon nanotubes

\begin{tabular}{ll}
\hline Parameters & Values \\
\hline Outside diameter & $<8 \mathrm{~nm}$ \\
Inside diameter & $2 \sim 5 \mathrm{~nm}$ \\
-COOH content & $3.86 \mathrm{wt} \%$ \\
Length & $10 \sim 30 \mu \mathrm{m}$ \\
Purity & $>95 \%$ \\
Ash & $<1.5 \mathrm{wt} \%$ \\
Special surface area & $>500 \mathrm{~m}^{2} / \mathrm{g}$ \\
Electrical conductivity & $>10^{2} \mathrm{~s} / \mathrm{cm}^{3}$ \\
Density & $\sim 2.1 \mathrm{~g} / \mathrm{cm}^{3}$
\end{tabular}

vibrator was used to compact the samples. The samples were then surface-smoothed, and covered with plastic films. All samples were demolded $24 \mathrm{~h}$ after casting. They were then cured for 28 days at a temperature of $20^{\circ} \mathrm{C}$ and a relative humidity of $100 \%$. All samples were dried at a temperature of $50^{\circ} \mathrm{C}$ for 5 days before testing.

\section{MEASUREMENT}

Electrical resistance was measured by using a two-electrode method with a digital multimeter (Keithley 2100, Keithley Instruments Inc., USA) (Han et al., 2007, 2009b, 2011a, 2012). Compressive loads were applied using a material testing machine (ATS 900, Applied Test Systems, Inc., USA). Both electrical resistance and compressive loads were automatically recorded into a computer. All the experiments were carried out at room temperature. The morphologies of CNT filled cement mortar composites were examined on a scan electron microscope (JSM-6490LV, JEOL Ltd., USA). At least three specimens of each type of composites were tested.

\section{RESULTS \\ PRESSURE-SENSITIVITY OF CNT FILLED CEMENT MORTAR COMPOSITES WITH SDS}

Figure 1 shows the change of the electrical resistance $R$ of CNT filled cement mortar composites with SDS and 1 wt.\% of MWNT under repeated compressive loading and impulsive loading. Figure 1A shows that the relationship between the electrical resistance of these composites and the compressive stress $\sigma$ is irregular under repeated compressive loading with amplitude of 2.5 MPa. It can be seen from Figure 1B that the electrical resistance of composites decreases upon loading and increases with unloading in every cycle under repeated compressive loading with amplitude of $10 \mathrm{MPa}$, and the maximum change of electrical resistance $\Delta R$ (i.e., $R-R_{0}$ where $R_{0}$ is the initial electrical resistance of sample) reaches about $300 \Omega$ when stress is $10 \mathrm{MPa}$. However, there is no good repeatability of the change of electrical resistance in each cycle. Figure 1C shows the electrical resistance decreases with compressive stress under impulsive loading on the whole, but some of the impulsive loadings do not cause obvious change in the electrical resistance of composites. These findings indicate that the CNT filled cement mortar composites with SDS and 1 wt.\% of MWNT have very poor pressure-sensitivity.
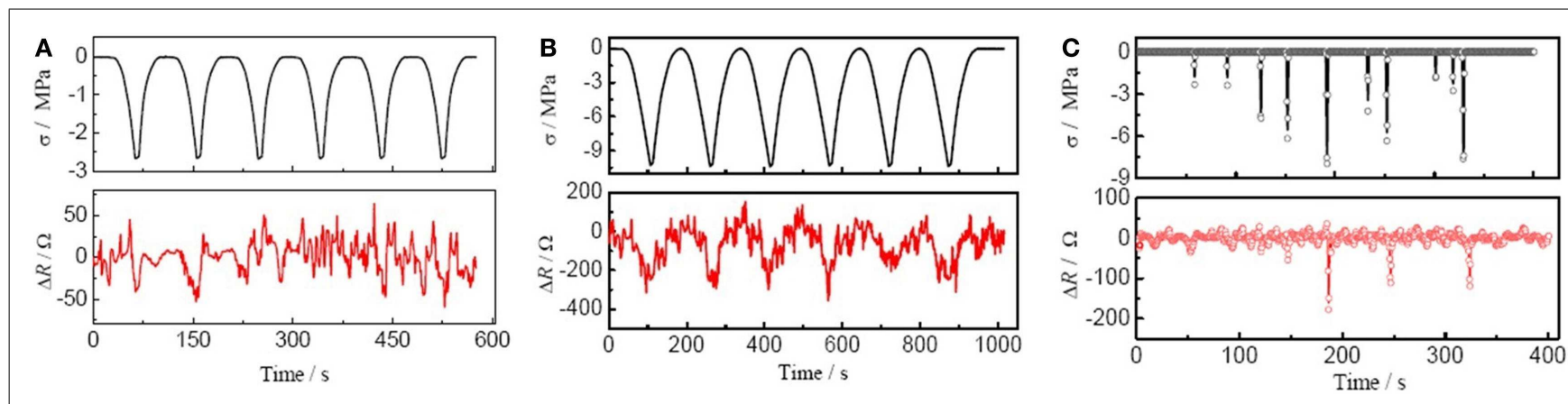

FIGURE 1 | Pressure-sensitivity of CNT filled cement mortar composites with SDS and 1 wt.\% of MWNT. (A) Under repeated compressive loading with amplitude of 2.5 MPa. (B) Under repeated compressive loading with amplitude of $10 \mathrm{MPa}$. (C) Under impulsive loading. 
Figure 2 gives that the relationships between compressive stress and the change in electrical resistance of CNT filled cement mortar composites with SDS and 0.2 wt.\% of MWNT under repeated compressive loading and impulsive loading. As shown in Figures 2A,B, the electrical resistance of composites decreases with loading and increases with unloading in every cycle under the repeated compressive loading with amplitudes of 2.5 and $10 \mathrm{MPa}$, and the change in electrical resistance respectively reaches about 120 and $300 \Omega$ maximum as the compressive stress is 2.5 and $10 \mathrm{MPa}$. However, the repeatability of the change of electrical resistance in each cycle is still not good, especially that under the repeated compressive loading with amplitude of 2.5 MPa. Figure 2C shows that most of the impulsive loadings can cause regular decrease of electrical resistance. A comparison of Figures 1 and $\mathbf{2}$ indicates that the CNT filled cement mortar composites with SDS and 0.2 wt.\% of MWNT have better pressure-sensitivity than those with SDS and $1 \mathrm{wt} . \%$ of MWNT, but the pressure-sensitivities of both them are unsatisfactory for real applications.

\section{PRESSURE-SENSITIVITY OF CNT FILLED CEMENT MORTAR COMPOSITES WITH NaDDBS}

The change in electrical resistance of CNT filled cement mortar composites with NaDDBS and 1 wt.\% of MWNT under repeated compressive loading and impulsive loading is illustrated in Figure 3. As shown in Figures $\mathbf{3 A}, \mathbf{B}$, the electrical resistance changes in same trends under the repeated compressive loads with amplitudes of 2.5 and $10 \mathrm{MPa}$. The change in electrical resistance decreases reversibly with loading and increases reversibly with unloading in every cycle, and it reaches about 360 and $700 \Omega$ as the compressive stress is 2.5 and $10 \mathrm{MPa}$, respectively. In addition, Figure 3C shows that there is a good corresponding relationship between the electrical resistance and the impulsive loading. These findings show that the CNT filled cement mortar composites with NaDDBS and $1 \mathrm{wt} . \%$ of MWNT have stable and good pressure-sensitivity.

Figure 4 shows that the response of electrical resistance of CNT filled cement mortar composites with NaDDBS and 0.2 wt.\% of MWNT to compressive stress when the sample was under repeated compressive loading and impulsive loading, respectively. By comparing Figures 3 and 4, we find that the change trend of electrical resistance of composites with NaDDBS and $0.2 \mathrm{wt} . \%$ of MWNT is similar to that of the composites with NaDDBS and $1 \mathrm{wt} . \%$ of MWNT under the repeated compressive loading of $10 \mathrm{MPa}$, but the electrical resistance change of the former only reaches $350 \Omega$. In addition, under the repeated compressive loading of $2.5 \mathrm{MPa}$, the repeatability of the change in electrical resistance is much worse than that of CNT filled cement mortar composites with NaDDBS and 1 wt.\% of MWNT. These findings indicate that the pressure-sensitivity of CNT filled cement mortar composites with
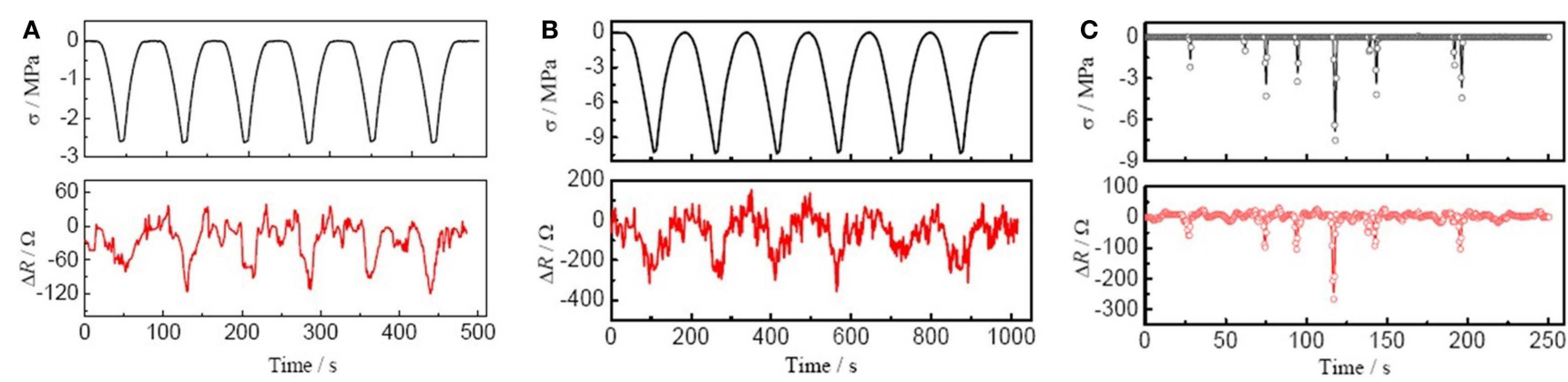

FIGURE 2 | Pressure-sensitivity of CNT filled cement mortar composites with SDS and $\mathbf{0 . 2}$ wt.\% of MWNT. (A) Under repeated compressive loading with amplitude of $2.5 \mathrm{MPa}$. (B) Under repeated compressive loading with amplitude of $10 \mathrm{MPa}$. (C) Under impulsive loading.
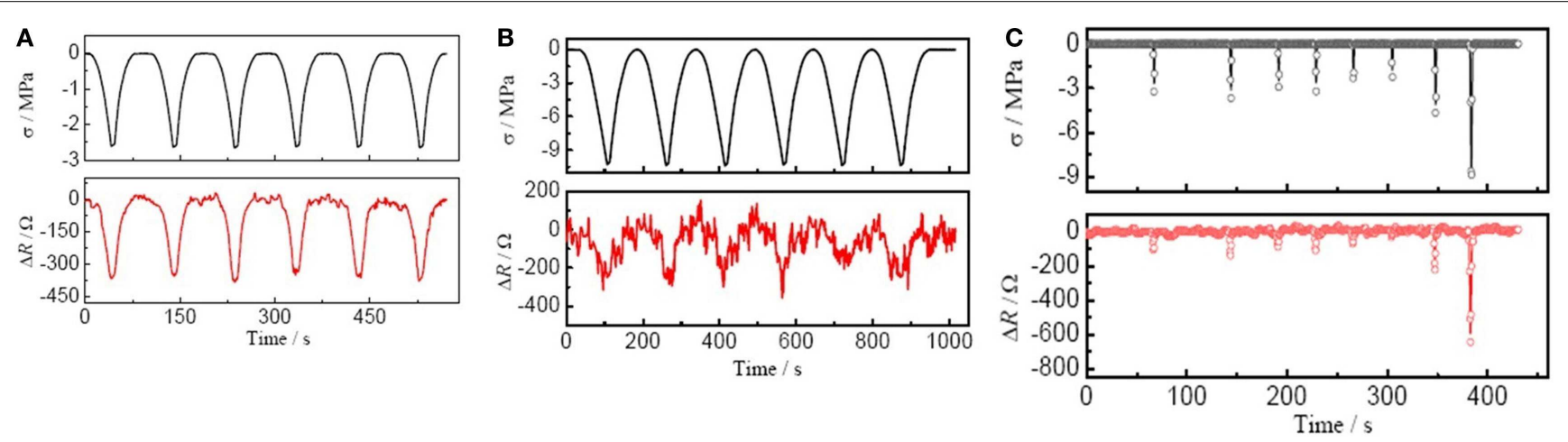

FIGURE 3 | Pressure-sensitivity of CNT filled cement mortar composites with NaDDBS and 1 wt.\% of MWNT. (A) Under repeated compressive loading with amplitude of $2.5 \mathrm{MPa}$. (B) Under repeated compressive loading with amplitude of $10 \mathrm{MPa}$. (C) Under impulsive loading. 

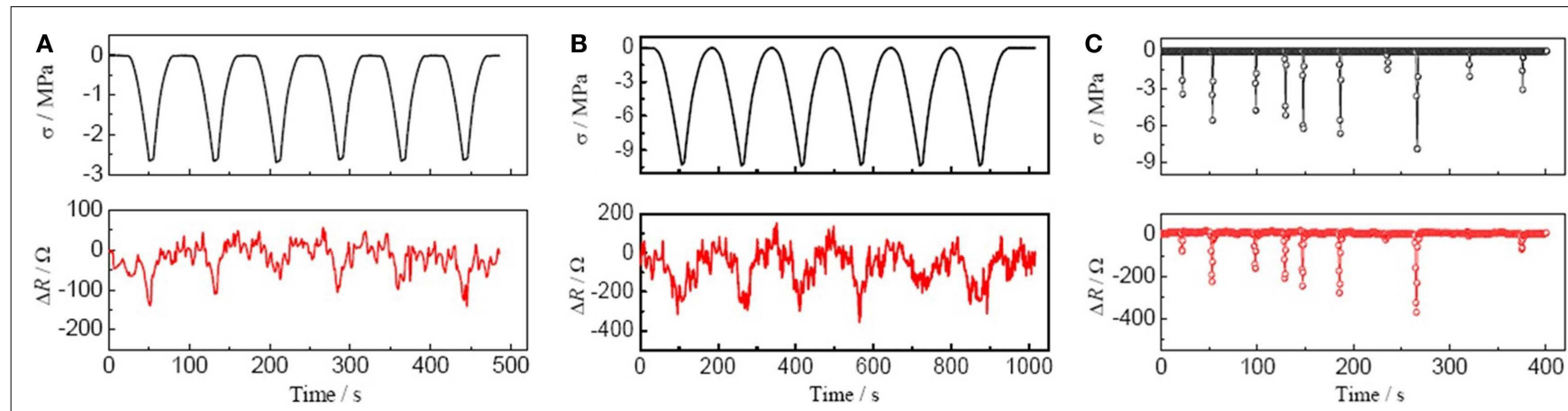

FIGURE 4 | Pressure-sensitivity of CNT filled cement mortar composites with NaDDBS and $\mathbf{0 . 2}$ wt.\% of MWNT. (A) Under repeated compressive loading with amplitude of $2.5 \mathrm{MPa}$. (B) Under repeated compressive loading with amplitude of $10 \mathrm{MPa}$. (C) Under impulsive loading.
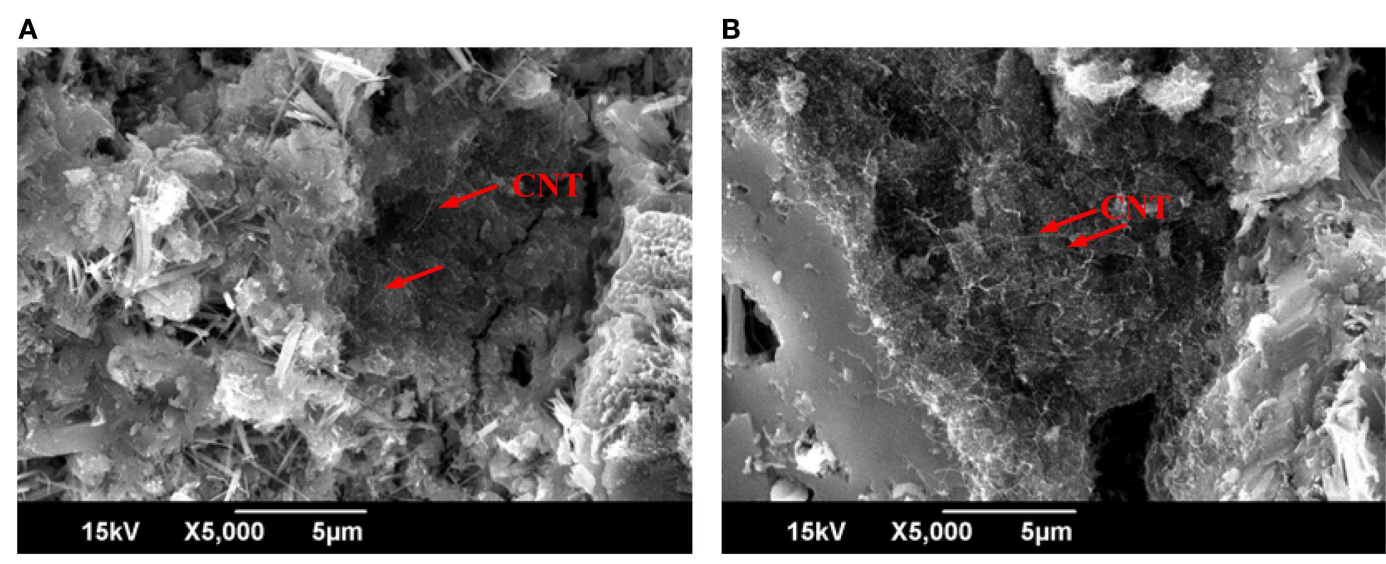

FIGURE 5 | SEM photographs of CNT filled cement mortar composites. (A) With NaDDBS and 1 wt. \% of MWNT. (B) With SDS and 1 wt. \% of MWNT.

NaDDBS and 0.2 wt.\% of MWNT is worse than that of the composites with NaDDBS and 1 wt.\% of MWNT. However, Figure 4C shows that this kind of composites also have stable and sensitive pressure-sensitive responses to impulsive loads.

\section{DISCUSSION ON MECHANISM}

The above experimental results show that the pressure-sensitive properties of CNT filled cement mortar composites are closely relative to the kinds of surfactants and the concentrations of MWNT. The repeatability and sensitivity (i.e., the change in electrical resistance per unit compressive stress) of pressure-sensitivity of the composites with NaDDBS and 1 wt.\% of MWNT, with NaDDBS and 0.2 wt.\% of MWNT, with SDS and 0.2 wt.\% of MWNT, and with SDS and 1 wt.\% of MWNT decrease orderly.

The pressure-sensitivity for CNT filled composites is caused by the following four reasons: (1) the electric conductivity of CNT varies under external force (Tombler et al., 2000); (2) the number of contact points of CNT increases with the increase of loading, which can cause an enhancement of conductivity; (3) the separation distance between CNT decreases under compressive loading, which can cause an enhancement in tunneling effect conduction; (4) the field induced tunneling effect enhances due to compressive loading. According to the Fowler-Nordheim theory, CNT have a strong field emission effect under electric field. The local high electrical field in composites increases the potential energy of electrons through tunneling barrier between CNT, which causes the enhancement in tunneling effect conduction (Meyyappan, 2005; Li et al., 2007; Han et al., 2011b). Furthermore, when the composites are deformed under compressive loading the separation between CNT will be reduced, i.e., the tunneling barrier to be transited by electrons will decrease and the field induced tunneling can more easily occur in the composites (Chen et al., 2004; Li et al., 2007). As a result, the repeatability and sensitivity of the pressure-sensitivity of CNT filled cement mortar composites are strongly influenced by the conductive network in composites, which in turn is decided by the dispersion of CNT in cement mortar. Therefore, it can be concluded that the difference between the pressure-sensitivities of CNT filled cement mortar composites with different surfactants and different concentrations of MWNT is due to the different dispersion of CNT in these composites.

The reasons why CNT filled cement mortar composites with NaDDBS have better pressure-sensitivity than those with SDS can be explained as follows. SDS has weaker interaction with nanotube surfaces than NaDDBS because it does not have a benzene ring 
like NaDDBS. The $\pi$-like stacking of the benzene rings of $\mathrm{NaD}$ DBS can interact with the surfaces of MWNTs via $\pi-\pi$ and $\mathrm{CH}-\pi$ interactions, which can significantly enhance the binding and surface coverage of surfactant molecules to graphite (Vaisman et al., 2006). As a result, the dispersing capability of NaDDBS to CNT is superior to that of SDS. Figure $\mathbf{5}$ shows the SEM photographs of two kinds of CNT filled cement mortar composites. It can be seen from Figure 5B that there are some aggregates of CNT in the composites with SDS and $1 \mathrm{wt} . \%$ of MWNT. Although the ratio of surfactant to CNT decreases with the increase of the concentration of CNT, the concentration of NaDDBS adopted in this study can disperse as high as $1.0 \mathrm{wt} . \%$ of MWNT owing to their superior dispersing capability to CNT. Therefore, the CNT filled cement mortar composites with NaDDBS and 1 wt.\% of MWNT presents better pressure-sensitivity than those with NaDDBS and 0.2 wt.\% of MWNT. On the contrary, the dispersing capability of SDS to CNT is weaker than that of NaDDBS, so the dispersing effect of SDS to $1 \mathrm{wt} . \%$ of MWNT is actually worse than that to $0.2 \mathrm{wt} . \%$ of MWNT. As a result, the CNT filled cement mortar composites with SDS and $0.2 \mathrm{wt} . \%$ of MWNT have better pressure-sensitivity than those with SDS and 1 wt.\% MWNT.

\section{CONCLUSION}

Two types of surfactants, SDS, and NaDDBS, were used to disperse MWNT in cement mortar and fabricate pressure-sensitive CNT filled cement mortar composites in this study. The change of electrical resistance of CNT filled cement mortar composites with different concentrations of MWNT and different surfactants was studied under compression and impact, respectively. The research results show that the repeatability and sensitivity of pressure-sensitivity of the CNT filled cement mortar composites with NaDDBS and 1 wt.\% of MWNT, with NaDDBS and 0.2 wt.\% of MWNT, with SDS and 0.2 wt. $\%$ of MWNT, and with SDS and 1 wt.\% of MWNT to external force decrease orderly. These findings indicate that NaDDBS has higher efficiency for the dispersion of MWNT in cement mortar than SDS, and it is an effective surfactant for fabricating MWNT filled cement-based composite with better pressure-sensitivity.

\section{ACKNOWLEDGMENTS}

The authors thank the funding supported from the National Science Foundation of China (grant Nos. 51428801, 51178148), the US National Science Foundation (CMMI-0856477), and Federal Highway Administration (FHWA) of US Department of Transportation (DTFH61-10-C-00011).

\section{REFERENCES}

Chen, B., Wu, K. R., and Yao, W. (2004). Conductivity of carbon fiber reinforced cement-based composites. Cement Concrete Compos. 26, 291-297. doi:10.1016/ S0958-9465(02)00138-5

Chung, D. D. L. (2002). Piezoresistive cement-based materials for strain sensing. J. Intell. Mater. Syst. Struct. 13, 599-609. doi:10.1106/104538902031861

Han, B., Yu, X., Kwon, E., and Ou, J. (2012). Effects of CNT concentration level and water/cement ratio on the piezoresistivity of CNT/cement composites. J. Compos. Mater. 46, 19-25. doi:10.1177/0021998311401114

Han, B., Yu, X., and Ou, J. (2011a). "Multifunctional and smart carbon nanotube reinforced cement-based materials," in Nanotechnology in Civil Infrastructure, eds
K. Gopalakrishnan, B. Birgisson, P. Taylor, and N. O. Attoh-Okine (Springer), 1-48. doi:10.1007/978-3-642-16657-0_1

Han, B. G., Han, B. Z., Yu, X., and Ou, J. P. (2011b). Ultrahigh pressure-sensitive effect induced by field emission at sharp nano-tips on the surface of spiky spherical nickel powders. Sens. Lett. 9, 1629-1635. doi:10.1166/sl.2011.1719

Han, B. G., Guan, X. C., and Ou, J. P. (2007). Electrode design, measuring method and data acquisition system of carbon fiber cement paste piezoresistive sensors. Sens. Actuators A-Phys. 135, 360-369. doi:10.1016/j.sna.2006.08.003

Han, B. G., Han, B. Z., and Ou, J. P. (2009a). Experimental study on use of metal powder-filled cement-based composite for fabrication of piezoresistive sensors with high sensitivity. Sens. Actuators A-Phys. 149, 51-55. doi:10.1016/j.sna.2008. 10.001

Han, B., Yu, X., and Kwon, E. (2009b). Self-sensing CNT/cement composite for traffic monitoring. Nanotechnology 20, 445501. doi:10.1088/0957-4484/20/44/445501

Han, B. G., and Ou, J. P. (2007). Embedded piezoresistive cement-based stress/strain sensor. Sens. Actuators A-Phys. 138, 294-298. doi:10.1016/j.sna.2007.05.011

Hou, T. C., and Lynch, J. P. (2005). Conductivity-based strain monitoring and damage characterization of fiber reinforced cementitious structural components. Proc. SPIE 5765, 7-10. doi:10.1117/12.599955

Islam, M. F., Rojas, E., Bergey, D. M., Johnson, A. T., and Yodh, A. G. (2003). High weight fraction surfactant solubilization of single-wall carbon nanotubes in water. Nano Lett. 3, 269-273. doi:10.1021/nl025924u

Li, G. Y., Wang, P. M., and Zhao, X. H. (2007). Pressure-sensitive properties and microstructure of carbon nanotube reinforced cement composites. Cement Concrete Compos. 29, 377-382. doi:10.1016/j.cemconcomp.2006.12.011

Mao, Q. Z., Chen, P. H., Zhao, B. Y., and Li, Z. Q. (1996). Resistance changement of compression sensible cement specimen under different stresses. J. Wuhan Univ. Technol. 11, 41-45.

Meyyappan, M. (2005). Carbon Nanotubes Science and Applications. Boca Raton: CRC Press.

Ou, J. P., and Han, B. G. (2009). Piezoresistive cement-based strain sensors and self-sensing concrete components. J. Intell. Mater. Syst. Struct. 20, 329-336. doi:10.1177/1045389X08094190

Tan, Y. Q., and Resasco, D. E. (2005). Dispersion of single-walled carbon nanotubes of narrow diameter distribution. J. Phys. Chem. B 109, 14454-14460. doi:10.1021/jp052217r

Tombler, T. W., Zhou, C., Alexseyev, L., Kong, J., Dai, H., Liu, L., et al. (2000). Reversible electromechanical characteristics of carbon nanotubes under localprobe amanipulation. Nature 405, 769-772. doi:10.1038/35015519

Vaisman, L., Wagner, H. D., and Marom, G. (2006). The role of surfactants in dispersion of carbon nanotubes. Adv. Colloid Interface Sci. 12, 37-46. doi:10.1016/ j.cis.2006.11.007

Yu, J. R., Grossiord, N., Koning, C. E., and Loos, J. (2007). Controlling the dispersion of multi-wall carbon nanotubes in aqueous surfactant solution. Carbon N. Y. 45, 618-623. doi:10.1016/j.carbon.2006.10.010

Yu, X., and Kwon, E. (2009). Carbon-nanotube/cement composite with piezoresistive property. Smart Mater. Struct. 18, 055010. doi:10.1088/0964-1726/18/5/ 055010

Conflict of Interest Statement: The Review Editor Xufeng Dong declares that, despite being affiliated with the same institution as author Baoguo Han, the review process was handled objectively and no conflict of interest exists. The authors declare that the research was conducted in the absence of any commercial or financial relationships that could be construed as a potential conflict of interest.

Received: 29 July 2014; accepted: 23 October 2014; published online: 06 November 2014.

Citation: Han B and Yu X (2014) Effect of surfactants on pressure-sensitivity of CNT filled cement mortar composites. Front. Mater. 1:27. doi: 10.3389/fmats.2014.00027

This article was submitted to Smart Materials, a section of the journal Frontiers in Materials.

Copyright (c) 2014 Han and Yu. This is an open-access article distributed under the terms of the Creative Commons Attribution License (CC BY). The use, distribution or reproduction in other forums is permitted, provided the original author(s) or licensor are credited and that the original publication in this journal is cited, in accordance with accepted academic practice. No use, distribution or reproduction is permitted which does not comply with these terms. 